\title{
Perspectives on Solid-State Flying Qubits
}

\author{
A. Bertoni \\ National Research Center on nanoStructures and bioSystems at Surfaces (S3), INFM-CNR \\ via Campi 213/A, 41100 Modena \\ e-mail: bertoni.andrea@unimore.it
}

\section{SOLID STATE FLYING QUBITS}

The quest for quantum-computing capable architectures has recently focused on solid-state implementations, since they seem more prone to meet the required criteria [1] of scalability and integrability than alternative approaches like the ones based on nuclear magnetic resonance, ion traps or quantum electrodynamics techniques [2].

In many proposals different components of a quantum-computing system are dedicated to different quantum transformations. As a consequence at each stage the outputs of these transformations, i.e. the state of a qubit register, must be moved to the inputs of other quantum gates. This process, overlooked in the early times of quantum information processing research, results to be critical in viable practical realizations [3], so that the celebrated Di Vincenzo checklist has been recently extended with two extra issues [4], namely (a) the ability to interconvert stationary and flying qubits, and (b) the ability to faithfully transmit flying qubits between specific locations. However these new requirements are redundant for proposals in which the logical operation is performed on the flying qubit itself. The present contribution will review the recent efforts towards the numerical modeling (and eventually the experimental realization) of prototypes of one- and two-qubit quantum gates (Fig. 1) based on electron transport in single and coupled quantum wires (QWRs) [5].

\section{ELECTRON STATES IN QUANTUM WIRES}

Two alternative possibilities for the qubit definition have been considered in literature: the charge localization of electrons transmitted through a couple of QWRs [6] (or, equivalently, the current states in an electron interferometer [7]), and the spin orientation of the carriers [8]. In both cases the theoretical feasibility of a universal set of quantum gates has been demonstrated. Furthermore it has been proposed to use surface acoustic waves as a highly controllable mean to inject and drive electrons in the QWRs [8,9]. We showed by numerical simulations that the introduction of the new time-dependent potential, while altering the dynamics of the quantum gates, preserves the logical transformation on the qubit [10] (Fig. 2).

\section{ENTANGLEMENT AND DECOHERENCE}

Quantum entanglement is the key-resource for quantum information processing but it represents a detriment when it couples the qubit state with some "external" degree of freedom (environment), leading to the well-known phenomenon of decoherence. If the modeling of the qubit system includes the quantum state of the environment, the quantitative evaluation of entanglement dynamics (and consequently of decoherence) can be obtained (Fig. 3), together with the few-particle effects originating from quantum correlation. A numerical approach will be presented to include few-particle dynamics in the calculation of scattering states in the framework of coherent transport through a QWR with localized states.

\section{REFERENCES}

[1] D. DiVincenzo, The physical implementation of quantum computation, quant-ph/0002077 (2000).

[2] J. A. Jones et al., J. Mag. Res. 135, 353 (1998).

L.M.K. Vandersypen et al., Nature 414, 883 (2001). C. A. Sackett et al., Nature 404, 256 (2000). V. Giovannetti et al., Phys. Rev. A 62, 32306 (2000).

[3] D. V. Khveshchenko, Phys. Rev. B 70, 92407 (2004). Y. L. Lim et al., Phys. Rev. A 73, 12304 (2006).

[4] Quantum Computation Roadmap, ARDA, http://qist.lanl.gov (2004).

[5] A. Bertoni et al., Phys. Rev. Lett. 84, 5912 (2000). R. Ionicioiu et al., Int. J. Mod. Phys. B15, 125 (2001) quant$\mathrm{ph} / 9907043$.

[6] A. Bertoni et al., J. Mod. Optics 49, 1219 (2002).

[7] T. Zibold at al., Proc. $14^{\text {th }}$ HCIS Conference, to appear (2006).

[8] C.H.W. Barnes et al., Phys. Rev. B 62, 8410 (2000).

[9] R. Rodriquez et al., Phys. Rev. B 72, 085329 (2005).

[10] A. Bertoni and al., Proc. 32nd ESSDERC Conf. (2002). 

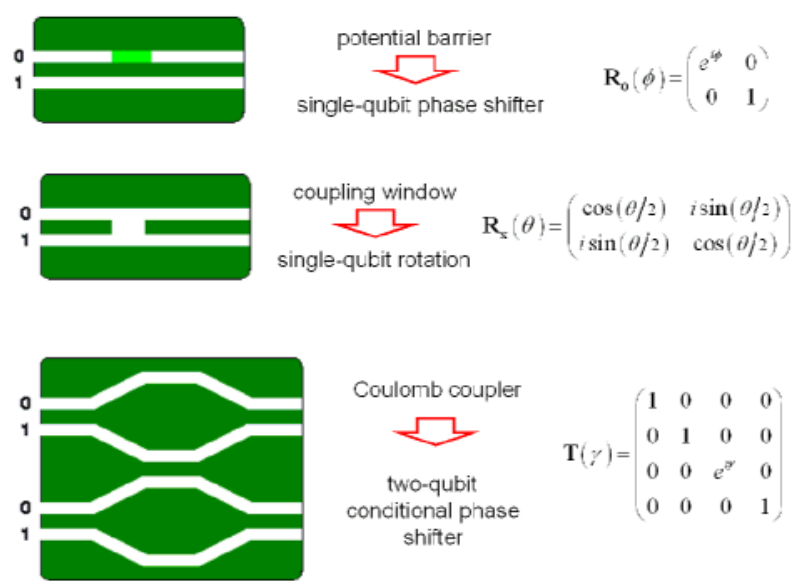

Coulomb coupler

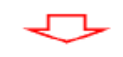

two-qubit concitional phase shitter

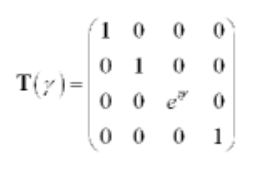

Fig. 1. Schematic representation of a universal set of quantum gates. [6]
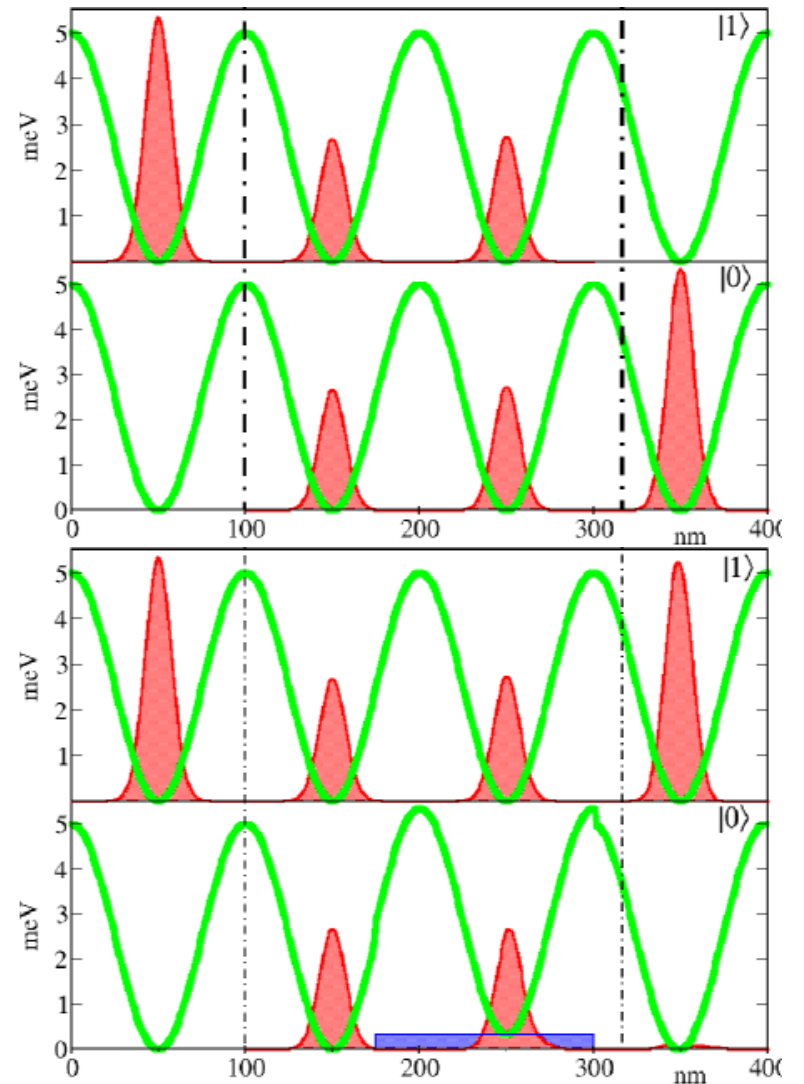

Fig. 2. One-dimensional time-dependent simulation of an electron wave function (red) trapped inside a minimum a surface acoustic wave (green) propagating (from the left to the right) along a coupled-wire device with two single-qubit rotations $R_{x}(\rho / 2)$ (represented by dashed-dotted lines). In the lower graph a phase shifter (blue) is inserted in the wire 0 between the rotations, leading to a different final state of the qubit. [10]

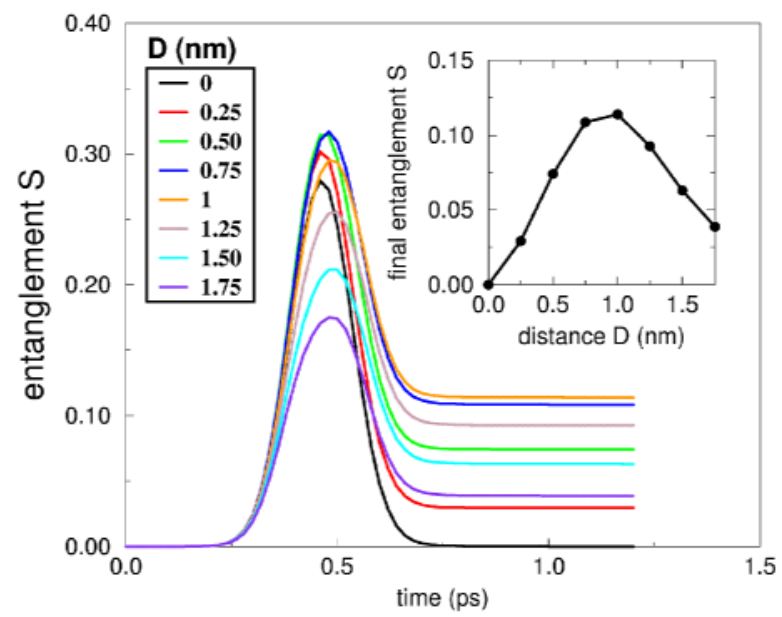

Fig. 3. Entanglement, as a function of time, of two electrons propagating in opposite directions along two parallel singlemode quantum wires. $\mathrm{D}$ represents the distance between the wires. The entanglement is computed from the von Neumann entropy $S(\rho)=-\operatorname{Tr}_{1}\left(\left(\operatorname{Tr}_{2} \rho\right) \log _{2}\left(\operatorname{Tr}_{2} \rho\right)\right)$, where $\rho$ is the two-particle densisty matrix and $T r_{\mathrm{n}}$ is the trace operation on the position variable of particle $n$. The inset show that the dependence on $\mathrm{D}$ of the final value of $\mathrm{S}$ is non monotonic. 